Előd Biszak ${ }^{1}$, Gábor Timár ${ }^{2}$

\title{
First vector components in the MAPIRE: 3D building modelling and Virtual Reality view
}

Summary: MAPIRE is an originally raster-based portal, presenting scanned and geo-referred historical topographic, cadastral and town maps, as overlays on modern cartographic datasets, such as OpenStreetMaps or HereMaps. Its first 3D option was adopted via the SRTM elevation dataset, still on raster base, showing 3D panoramas from the viewpoint, using the historical map layer. Here we introduce the first vector component of the MAPIRE, developed in Cesium software environment. To improve the city and town maps, we applied a new 3D building modelling utility (MapBox Vector Tiles). The details of the historical buildings - varying in different time layers - are reconstructed from archive plans as well as photographic heritage provided by the Arcanum (the host of MAPIRE) and historical postcards. Unrevealed building parts are defined according to the 'usual outline' of the given age. Using this technology, we are able to provide virtual 3D tours for the users of MAPIRE, first in the frame of the 'Budapest Time Machine' project. Besides, the Virtual Reality (VR) tour is available also in the service, still in raster basis: as a pilot project, we can follow pre-recorded paths on the historical map using VR glasses.

\footnotetext{
${ }^{1}$ Arcanum Database Ltd., Budapest, Hungary

${ }^{2}$ Deptartment of Geophysics and Space Science, ELTE Eötvös Loránd University, Budapest, Hungary [timar@caesar.elte.hu]
} 